\title{
Innate immunity mediated by dendritic cells/macrophages plays a central role in the early period in tumor treatment using gene of Mycobacterium tuberculosis antigen
}

\author{
Takahiro USHIGUSA ${ }^{1,2)}$, Yoshiyuki KOYAMA ${ }^{1,3)}$, Tomoko ITO ${ }^{1,3)}$, \\ Kenichi WATANABE ${ }^{4}$, James K. CHAMBERS ${ }^{4)}$, Aya HASEGAWA ${ }^{1)}$,

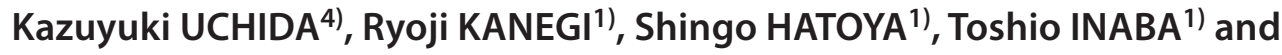 \\ Kikuya SUGIURA ${ }^{1) *}$
1) Department of Advanced Pathobiology, Graduate School of Life and Environmental Sciences, Osaka Prefecture University, Izumisano, Osaka 598-8531 Japan
${ }^{2)}$ Animal Regerative Medicine Center Hospital, Naka-ku, Yokohama, Kanagawa 231-0033 Japan
3) Japan Anti-tuberculosis Association, Shin-Yamanote Hospital, Higashimurayama, Tokyo 189-0021 Japan
${ }^{4)}$ Department of Veterinary Pathology, Graduate School of Agricultural and Life Sciences, The University of Tokyo, Bunyo-ku, Tokyo 113-5657, Japan

\section{J. Vet. Med. Sci.}

80(2): 190-196, 2018

doi: 10.1292/jvms.17-0466

Received: 21 August 2017

Accepted: 14 December 2017 Published online in J-STAGE:

1 January 2018

\begin{abstract}
By using a complex of DNA, polyethylenimine and chondroitin sulfate, the in vivo transfection of early secretory antigenic target-6 (ESAT-6) gene into tumor cells was found to cause significant suppression of the tumor growth. In order to apply the method in clinical cancer treatment in dogs and cats, mechanisms underlying the suppressive effects were investigated in a tumor-bearing mouse model. The transfection efficiency was only about $10 \%$, but the transfection of ESAT-6 DNA nevertheless induced systemic immune responses against ESAT-6. By triple injection of ESAT-6 DNA at three day intervals, the tumor was significantly reduced and almost disappeared by 5 days after the start of treatment, and did not increase for more than 15 days after the final treatment. In the immunohistochemistry, a larger number of dendritic cells (DCs)/macrophages expressing ionized calcium-binding adapter molecule 1 and $C D 3^{+} T$ cells was observed in tumors treated with ESAT-6 DNA, and their population further increased significantly by day 5 . Moreover, the amount of tumor necrosis factor, which is an apoptosis-inducing factor produced mainly by DCs/macrophages, was greater in the ESAT-6 DNA treated tumors than in controls, and increased with repeat of the treatment. These results indicate that in vivo transfection of ESAT-6 DNA into tumor cells elicits significant inhibition of tumor growth by inducing potent activity of innate immunity mediated by DCs/macrophages, which may be followed by adaptive immunity against tumor associated antigens, elicited by the costimulation with ESAT- 6 antigen.

KEY WORDS: dendritic cells/macrophages, early secretory antigenic target-6, gene therapy, innate immunity, tumor
\end{abstract}

\begin{abstract}
Although immunotherapy is a relatively new approach for treating tumors, it has already become an important part of treatment of many cancers. For an immune response to take place, a stimulating molecule must be recognized as a foreign object. Tumorassociated antigens (TAAs) appearing in tumor cells are believed to be appropriate targets for anti-cancer immunotherapy, and have been used in immunotherapeutic strategies against tumors. Tumor antigens are broadly classified into two categories according to their patterns of expression: shared non-mutated self-antigens that are overexpressed in cancer cells relative to normal cells, and tumor specific mutated antigens, so-called neoantigens. Preclinical and clinical evidence increasingly suggests an important role of mutant neoantigens in antitumor immunity [15]. These are expressed only on tumor tissues, so that T cells against the neoantigens are not subject to central immunological tolerance. In most cases, however, tumors have only non-mutated self-antigens, and lack expression of neoantigens capable of generating effective immunity. In such patients, defect in neoantigens on tumor cells impairs
\end{abstract}

*Correspondence to: Sugiura, K.: sugiura@vet.osakafu-u.ac.jp

O2018 The Japanese Society of Veterinary Science

This is an open-access article distributed under the terms of the Creative Commons Attribution Non-Commercial No Derivatives (by-nc-nd) License. (CC-BY-NC-ND 4.0: https://creativecommons.org/licenses/by-nc-nd/4.o/) 
the efficacy of immunotherapy.

To treat tumors not presenting neoantigens, we have developed a novel "artificial neoantigen strategy". In this strategy, tumor cells are transformed so as to express highly immunogenic antigens from pathogenic microorganisms as "artificial neoantigens", by means of in vivo transfection with plasmid DNA complexes [7, 21]. In a previous study, we chose "early secretory antigenic target-6" (ESAT-6), an antigen of Mycobacterium tuberculosis, as the artificial neoantigen [7]. ESAT-6 has unique properties which may promote immune-stimulation as "exogenous danger signals". ESAT-6 includes epitopes having high affinity for major histocompatibility complex (MHC) Class I [14] and Class II [12]. ESAT-6 has been shown to directly bind to Toll-like receptor 2 (TLR-2) [13]. ESAT-6-binding to TLR-2 stimulates macrophage activation and the secretion of tumor necrosis factor-alpha (TNF $\alpha$ ) and monocyte chemotactic protein-1 [10]. ESAT-6 induces interleukin (IL)- 6 and transforming growth factor-beta production in dendritic cells (DCs) through interaction with TLR-2, and generates an environment that promotes the differentiation of T helper-17 cells [1]. ESAT-6 could also induce differentiation of DCs from bone marrow. During the course of differentiation, proinflammatory cytokines such as TNF $\alpha$, interferon-gamma (IFN $\gamma$ ) and IL-12 were secreted from the DCs [9].

In the previous study [7], in order to examine the antitumor efficacy of ESAT-6 gene transfection, we injected intratumorally with a plasmid coding for the microbial antigen into tumor-bearing animals. High antitumor efficacy was observed, although the detailed mechanism is not clear. Antitumor effects should not be caused by the cytotoxicity of ESAT- 6 or the transfection reagents themselves, because transfection of the cells with the ESAT-6 gene caused only slight toxicity and cell death in vitro.

In the present study, the mechanism of antitumor effects induced by transfection of the "artificial neoantigen" was studied by observing an accumulation of immune cells in the tumor tissue, and measuring the cytokine levels in the tumor after the ESAT- 6 gene transfection.

\section{MATERIALS AND METHODS}

\section{Animals and tumor cell line}

The study protocols were approved by the animal experiment committee of Osaka Prefecture University (approval \#28-23). C57BL/6 (B6) mice were purchased from Japan SLC Inc. (Hamamatsu, Japan). The mice were maintained under specific pathogenfree conditions. They were euthanized if the tumor mass grew greater than $20 \mathrm{~mm}$, by anesthesia with sodium pentobarbital $(200 \mathrm{mg} / \mathrm{Kg}$, intraperitoneal injection) or by isoflurane followed by cervical dislocation. The B6-derived melanoma line B16 was obtained from the RIKEN BioResource Center (Tsukuba, Japan) and maintained in RPMI 1640 supplemented with 10\% FBS, $100 \mathrm{U} / \mathrm{m} l$ penicillin, and $100 \mu \mathrm{g} / \mathrm{m} l$ streptomycin (henceforth, RPMI medium).

\section{Preparation of DNA and vehicle for in vivo transfection}

As DNA to be transfected, we prepared expression plasmid, pcDNA 3.1/myc-His $(+)$ with ESAT-6 DNA or cDNA of green fluorescent protein (GFP). For in vivo transfection, the vehicle was constructed as described by Koyama et al. [5, 7], by mixing the plasmid DNA with chondroitin sulfate sodium salt (CS: Seikagaku Corp., Tokyo, Japan) and polyethylenimine "Max" (PEI: Polyscience, Inc., Warrington, PA, U.S.A.): the DNA/CS/PEI complex.

\section{Treatment of mice}

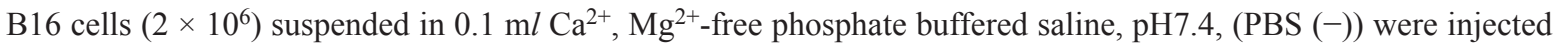
subcutaneously into the backs of B6 mice. Treatments began 5 days after the injection, when the diameter of the tumors was 5-6 mm (day 0). To determine the transfection efficiency, the GFP-DNA/CS/PEI complex containing $100 \mu \mathrm{g}$ DNA was injected into the tumors on day 0 . After $48 \mathrm{hr}$, the mice were euthanized with sodium pentobarbital followed by cervical dislocation, and the tumors were collected. To determine the immune response against ESAT-6 antigen, the ESAT-6-DNA/CS/PEI complex containing $100 \mu \mathrm{g}$ DNA was injected into the tumor on day 0 and day 3. Peripheral blood of the mice was collected to determine the response against ESAT- 6 on day 5 from the vena cava under anesthesia with isoflurane, using a heparin-treated 23G-needle and a $1 \mathrm{ml}$-syringe. In the experiments on tumor growth, the ESAT-6-DNA/CS/PEI complex containing $100 \mu \mathrm{g}$ DNA was injected into the tumor on day 0 , day 3 and day 6 . The tumor size was measured 2-3 times per week. The tumor volume $\left(\mathrm{cm}^{3}\right)$ was calculated according to the formula: (longest diameter) $\times($ short diameter $) \times($ short diameter $) \times 0.5$. In the immunohistochemistry, the ESAT-6DNA/CS/PEI was injected into the tumor on day 0 and day 3. The mice were euthanized on day 1 or day 5 to collect tumor. In the experiments on cytokine production in tumors, the ESAT-6-DNA/CS/PEI was injected every other day. Tumors were collected at the day after second or fifth injection, to study the typical status in the early phase after treatments. For all experiments, as controls, PBS was injected into tumors the same as the ESAT-6-DNA/CS/PEI.

\section{Examination of transfection efficiency}

The transfection efficiency was determined by counting the GFP-expressing cells against all cells in the field of the frozen sections, using confocal laser microscopy (EZ-C1 3.90, Nikon Corp., Tokyo, Japan). Experiments were performed using three mice in each treatment group. Three sections were made from the tumor of each mouse. The cells in three or four high-power $(\times 400)$ fields were counted in each section. A total of more than 1,000 cells was counted for each tumor.

\section{Examination of immune response against ESAT-6 antigen}

Peripheral blood $(0.2 \mathrm{~m} l)$ was collected from the treated mice and incubated in tubes in the presence or absence of antigens of 
Mycobacterium tuberculosis including ESAT-6 (QuantiFERON ${ }^{\circledR}$ TG Gold, Japan BCG, Tokyo, Japan). Following overnight incubation at $37^{\circ} \mathrm{C}$, plasma was collected after centrifugation at $2,000 \mathrm{~g}$ for $15 \mathrm{~min}$. As immune responses, IFN $\gamma$ in the plasmas was measured using a mouse IFN $\gamma$ Quantikine ELISA Kit (R\&D Systems, Inc., MN, U.S.A.).

\section{Immunohistochemistry}

Immunohistochemistry were performed as described previously [18], using rabbit plyclonal antibodies against ionized calcium-binding adapter molecule 1 (Iba1: Wako, Osaka, Japan), which is a marker of macrophage and DCs $[2,8]$, human CD3 (Dako, Tokyo, Japan), human CD20 (Thermo Fisher Scientific, Waltham, MA, U.S.A.) and human granzyme B (Spring Bioscience, Pleasanton, CA, U.S.A.), which is a marker of cytotoxic lymphocytes (CTLs) and natural killer (NK) cells [17]. Cross reactivity of these antibodies to the corresponding mouse antigens was confirmed by the manufacturers. Experiments were performed using four mice in each treatment group. The numbers of positive cells were counted in five high-power $(\times 400)$ fields selected randomly. Results were expressed as the mean for the four tumors.

\section{Detection of cytokines in tumors}

The concentrations of TNF $\alpha$, IFN $\gamma$, IL-12, IL- 6 and IL-10 in lysate of tumors were determined as reported by Cook et al. [3] using a detection kit (BD Cytometric Bead Array (CBA) Mouse Inflammation Kit, Becton Dickinson Biosciences, San Jose, CA, U.S.A.) and a flow cytometer (FACS Canto II, Becton Dickinson Biosciences). The lysate was prepared by homogenizing the tumor tissue in a $1.5 \mathrm{ml}$-microtube, followed by centrifugation.

\section{Statistics}

Comparison of more than three parameters was performed by 1-way analysis of variance followed by the Tukey-Kramer test. Student's $t$ test for unpaired samples was used for comparison of two parameters. Differences between groups were taking as significant at $P<0.05$.
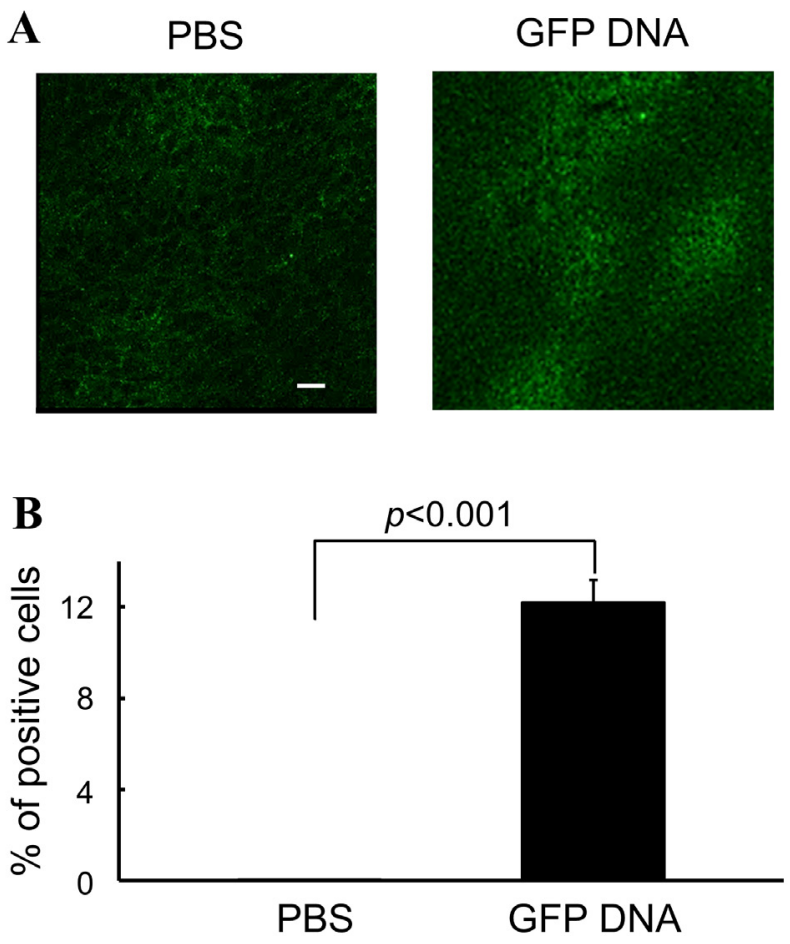

Fig. 1. Transfection efficiency of the DNA/PEI/CS complex. The GFP-DNA/PEI/CS complex was injected into tumors of the B16 melanoma cells growing in B6 mice. Two days after injection, tumors were collected and tested for transfection efficiency. As a control, PBS was injected into tumors. Appearance of GFP expression on fluorescence microscopy. A bar in the figure is $10 \mu \mathrm{m}$. (A), and the efficiency of transfection by the DNA/PEI/ CS complex (B) is shown. Experiments were performed using 3 mice in each group. The result is expressed as mean \pm SEM. Parameters were compared using Student's $t$ test.

\section{RESULTS}

\section{Transfection efficiency of the DNA vehicle into tumor cells}

The transfection efficiency of the DNA/CS/PEI complex was investigated before the examination of its therapeutic effect. As it was found that the expression of transfected gene was maximal at $48 \mathrm{hr}$ after injection the DNA/CS/PEI complex [6], the efficiency was studied using the tumor samples collected at $48 \mathrm{hr}$ after injection. As shown in Fig. 1, the transfection efficiency of the DNA vehicle into B16 cells was $12.3 \%$.

\section{Significant enhancement of ESAT-6 specific immune response by the ESAT-6 DNA treatment}

To examine whether the transfected ESAT-6 DNA induces a specific immune response against the corresponding antigen, peripheral blood was collected from the treated mice and the response against ESAT-6 antigen was determined. As shown in Fig. 2, the response of peripheral blood cells to ESAT-6 antigen was significantly enhanced in the ESAT-6 DNA group.

\section{Significant suppression of tumor growth by the ESAT-6 DNA treatment}

As shown in Fig. 3, B16 tumors in the control group injected with PBS immediately and significantly increased in size, and reached the chosen end point no longer than 18 days after the start of treatment. In contrast, the tumors in the ESAT-6 DNA treated group were almost completely suppressed for 21 days, and increased gradually thereafter, but did not reach the end point before 45 days after the start of treatment.

\section{Effect of the ESAT-6 DNA treatment on infiltration of immune cells}

The dynamic state of the infiltrated cells was studied using tumors collected either one day after first treatment (day 1) or two 


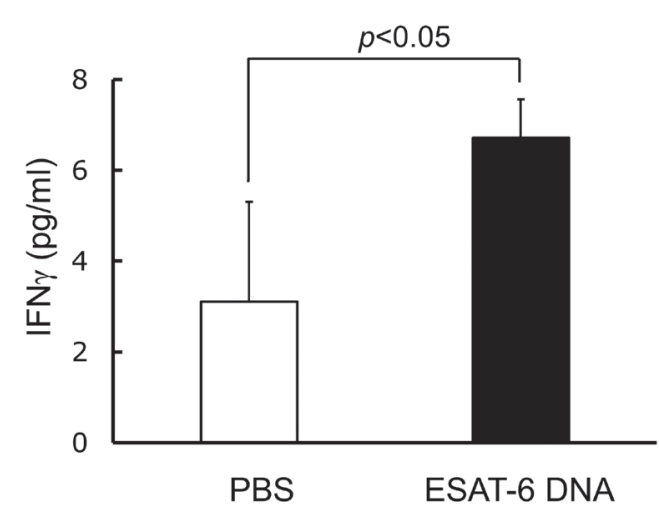

Fig. 2. Effect of ESAT-6 DNA transfection on the immune response against ESAT-6 antigen. ESAT6-DNA/CS/PEI complex or PBS was injected into tumors on day 0 and day 3 . Peripheral blood was collected from injected mice on day 5 . Responses of the blood cells against the ESAT-6 antigen was examined by measuring the IFN $\gamma$ produced. Closed bars show results for the group injected with the ESAT-6-DNA/PEI/CS complex. Experiments were performed using 4 mice in each group. Results are expressed as mean \pm SEM. Parameters were compared using Student's $t$ test.

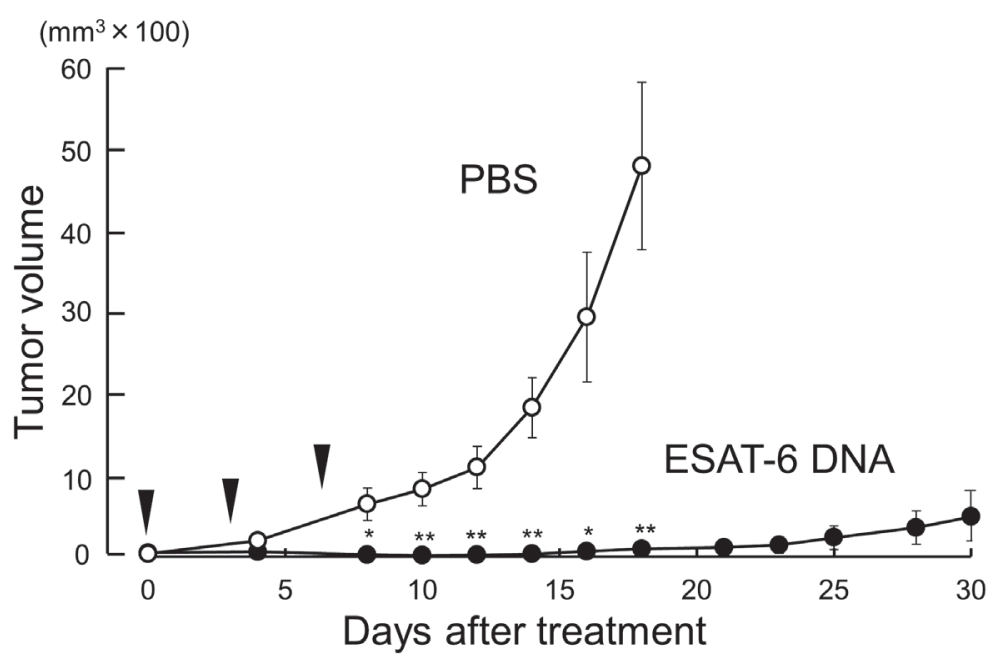

Fig. 3. Effect of ESAT-6 DNA transfection on tumor growth. The ESAT-6-DNA/ $\mathrm{PEI} / \mathrm{CS}$ complex or PBS was injected into B16 tumors in B6 mice on day 0, day 3 and day 6 (indicated by arrowhead). The tumor size was measured after the start of the treatments. Experiments were performed using 5 mice in each group. The result is expressed as mean \pm SEM. ${ }^{*} P<0.05, * * P<0.01$ vs PBS. Parameters were compared using Student's $t$-test.

\section{Iba-1}

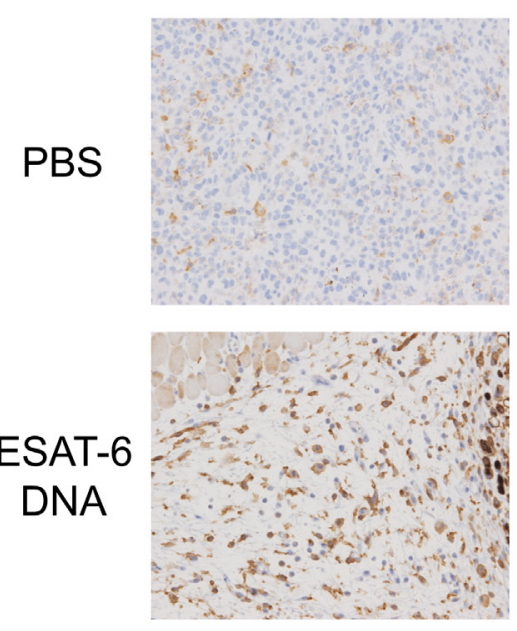

CD3
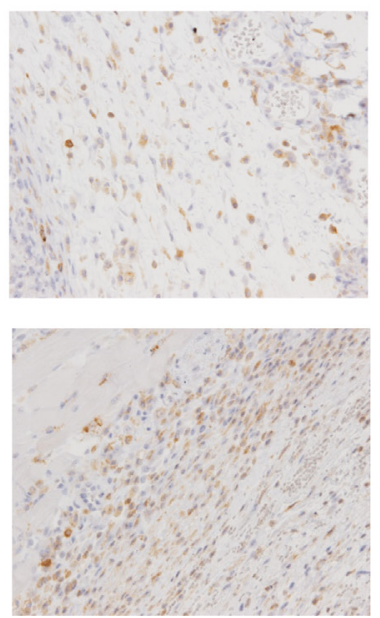

Granzyme B
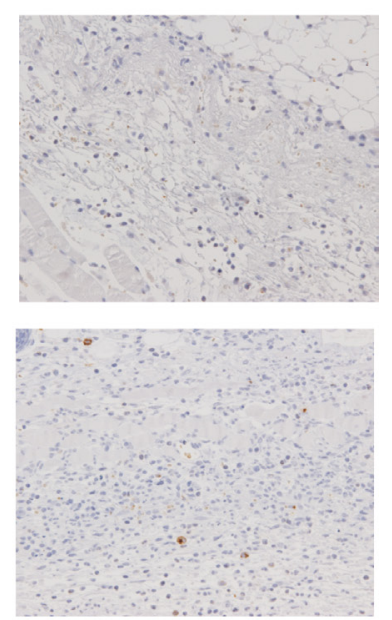

Fig. 4. Effect of ESAT-6 DNA transfection on the infiltration of immune cells in the tumor. The ESAT-6-DNA/CS/PEI complex or PBS was injected into the tumors on day 0 and day 3. Tumors were collected on day 1 or day 5 and were used for immunohistochemical staining using antibodies against the indicated immune cell marker. Representative results are shown for the day 5 tumor.

days after the second treatment (day 5). Representative microphotographs and numbers of the staining positive cells are shown in Figs. 4 and 6, respectively. As shown in Fig. 6A, the day 1 tumors did not differ in size between the ESAT-6 DNA-treated group and the PBS-treated group. However, the day 5 tumor of the ESAT-6 DNA group was significantly smaller than the day 1 tumors of the same group, whereas the day 5 tumors of the PBS group were larger $(P<0.1)$ than the day 1 tumors of the same group. In contrast to the results in tumor size, infiltration of cells expressing Iba1 in the day 5 tumors of the ESAT-6 DNA group was significantly greater than in either the day 1 tumors of the same group or the day 5 tumors of the PBS group (Figs. 4, 5 and 6B). The number of Iba $1^{+}$cells did not significantly differ between the day 5 and the day 1 tumors of the PBS group. It is noteworthy that in the tumor of the ESAT-6 DNA group, most of cells around $\mathrm{Iba}^{+}$cells had a shrunken nucleus with concentrated chromatin or crushing findings, which are characteristic of apoptosis (Fig. 5). As with the infiltration of $\mathrm{Iba}^{+}{ }^{+}$cells, infiltration of $\mathrm{CD} 3^{+} \mathrm{T}$ 
PBS

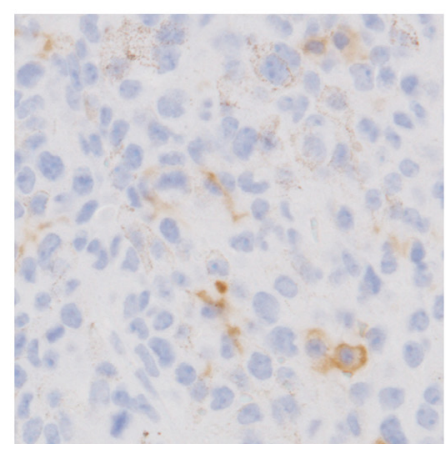

Fig. 5. Magnified pictures of the Iba-1staining in Fig. 4 were shown.
ESAT-6 DNA

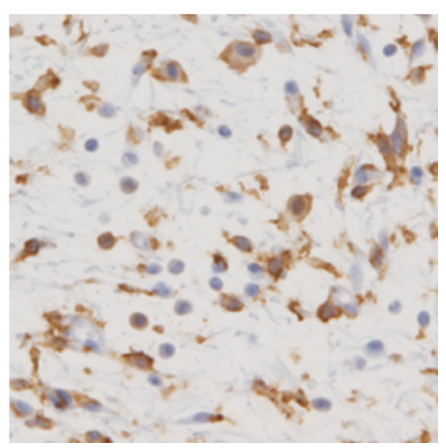

A
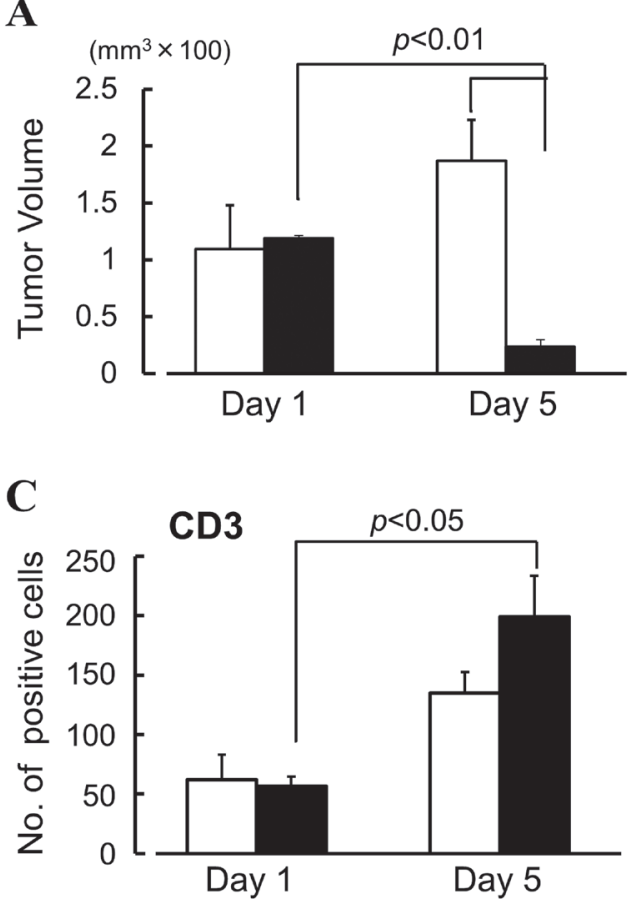
.
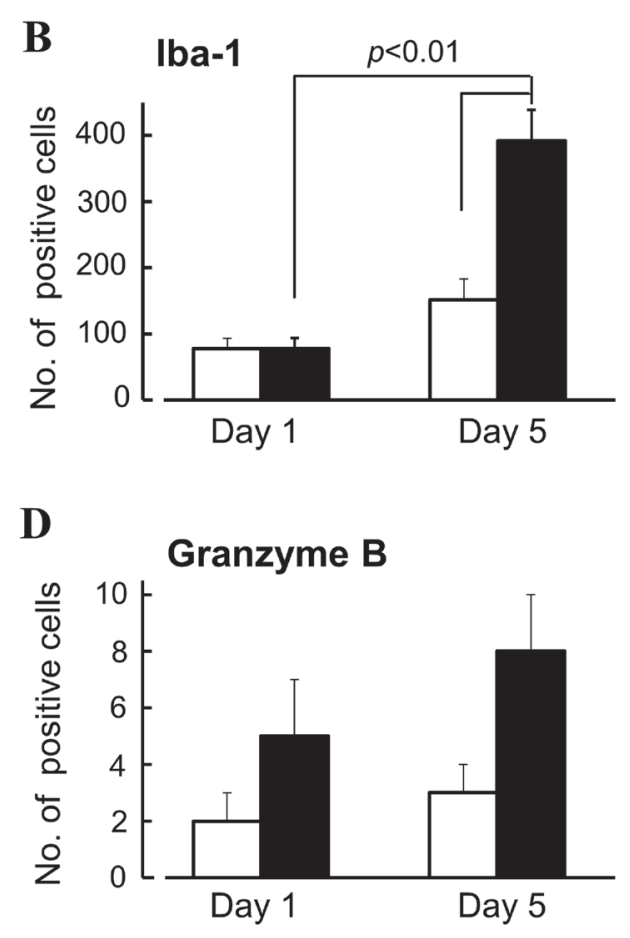

Fig. 6. Effect of ESAT-6 DNA transfection on the infiltration of immune cells in the tumor. The ESAT-6-DNA/CS/PEI complex or PBS was injected as described in the caption of Fig. 4, and the tumors were collected. The tumor volume (A) and results of immunohistochemical staining against the indicated antigens (B-D) are shown. Open bars show results for the PBS-injected group. Closed bars show results for the ESAT-6 DNA-injected group. Experiments were performed using 4 mice in each group. Results were expressed as mean \pm SEM. Parameters were compared using the Tukey-Kramer test.

cells in the day 5 tumors of the ESAT-6 DNA group was significantly greater than in the day 1 tumors of the same group (Figs. 4 and $6 \mathrm{C}$ ). But the number of $\mathrm{CD}^{+}$cells did not significantly differ between the day 5 and the day 1 tumors of the PBS group. The day 5 tumors of the ESAT-6 DNA group had more granzyme B-expressing cells than the day 1 tumors of the same group. However, the numbers were very small and therefore did not significantly differ (Figs. 4 and 6D). The infiltrated CD20 $0^{+}$cells were very few in all samples (data not shown).

\section{Effect of the ESAT-6 DNA treatment on cytokine production in tumors}

As shown in Fig. 7A, as with the number of Iba1+ cells, production of TNF $\alpha$ in the ESAT-6 DNA-treated tumors increased with a repeat of the treatment, and was always higher than in the PBS-treated tumors. Small amounts of IL-12 were observed in the later period in both the ESAT-6 DNA- and the PBS-treated tumors (Fig. 7B). IFN $\gamma$ was produced in the ESAT-6 DNA-treated tumors, but did not increase with a repeat of the treatment (Fig. 7C). Relative amount of IL-6 was detected in both the ESAT-6 DNA- and PBS-treated tumors. But the amount did not increase with repeating the treatments (Fig. 7D). 
A

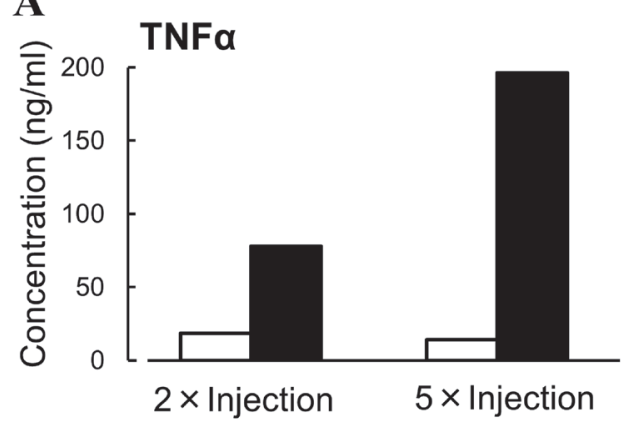

C

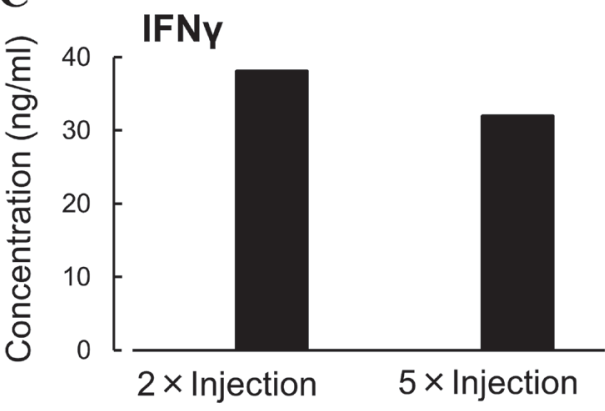

B

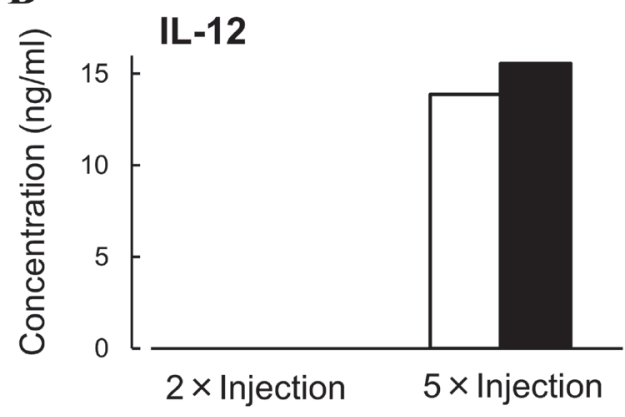

D

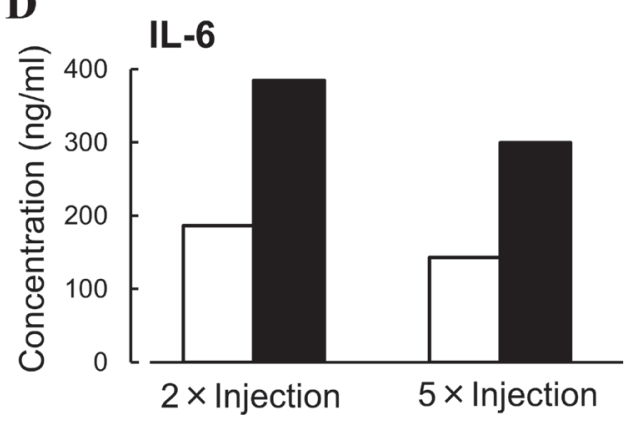

Fig. 7. Effect of ESAT-6 DNA transfection on cytokine production in the tumor. The ESAT-6-DNA/CS/PEI complex or PBS was injected every other day. The tumors were collected on the day after the second or fifth injection. The indicated cytokines in lysate of the tumors are quantified by flow cytometry as described in the MATERIALS AND METHODS section. The experiments were repeated and reproducible results were obtained. Results are expressed as means of two experiments.

\section{DISCUSSION}

In order to overcome the weak immunogenicity of tumor, studies were performed to induce expression of immunogenic antigens on tumor cells by transfecting genes so as to provide an "artificial neoantigen" such as adenovirus death protein or ESAT-6 [7, 21]. ESAT-6 epitope expressed by the ESAT-6-transfected tumor cells would trigger maturation of antigen presenting cells (APCs). The activated APCs are also expected to take up TAAs and elicit strong priming against the TAAs. In the present study, transfection of ESAT-6 DNA using the DNA/CS/PEI complex elicited the ESAT-6 specific immune responses and potent suppression of tumor growth as soon as 5 days after treatment (Figs. 2 and 6A). However, transfection efficiency of the transfection system was only around $10 \%$ (Fig. 2). These results suggest that the effect of immune response elicited by transfection of ESAT-6 DNA takes effect earlier and more effective than expected [4] if the responses were due merely to the mechanism of adaptive immunity described above.

Exosome, which is a highly efficient transporter, has been reported to be important in tumor antigen presentation [20] and activation of immune responses against tumor [19]. It also contributes to immune activation by antigens of M. tuberculosis [16]. We previously found that exosomes collected in vitro from B16 melanoma cells transfected with ESAT-6 DNA significantly enhanced immune responses not only against ESAT- 6 but also against untransfected B16 cells. Moreover, the exosome significantly suppressed the growth of the untransfected B16 tumor in vivo [5]. These observations imply that exosomes produced by the ESAT6 DNA-transfected tumor cells significantly enhance immune response against TAA as well as ESAT-6. This role of exosome may partially contribute to the higher efficiency in tumor suppression than expected from the low transfection efficiency.

In the immunohistochemistry, Iba $1^{+}$macrophages/DCs significantly increased by the ESAT-6 DNA treatment and solely correlated with the inhibition of tumor growth. Studies of the cytokine in treated tumors revealed that the production of TNF $\alpha$ correlates significantly with the number of $\mathrm{Iba}^{+}$cells infiltrated. This in turn is in inverse relationship with the volume of tumor. TNF $\alpha$ produced by activated DCs/macrophages is central to inducing apoptosis of tumors [11]. Indeed, many of cells observed around $\mathrm{Iba}^{+}$cells showed characteristic of apoptosis. ESAT-6 protein and its degraded peptides, as danger signals, have properties that induce activation of macrophages/DCs in a TLR-2-dependent or independent manner. These observations together indicated that the immediate decrease in tumor volume following the ESAT-6 DNA treatment is due to apoptosis which was mediated by innate immunity exerted by activated DCs/macrophages. Therefore, this mechanism is considered to be main explanation for the significant reduction of tumor in the early phase of the treatment though transfection efficiency was not so high.

The effect of the ESAT- 6 on the suppression of the tumor growth was maintained more than 15 days after the final treatment with ESAT-6 DNA. In addition, INF $\gamma$, which is a typical activator for Th1 immune response, was detected in the ESAT-6 DNA- 
treated tumors, but not in the PBS-treated tumors. In our previous study, exosomes derived from the ESAT-6 DNA transfected tumor cells also significantly enhanced immune responses against the TAAs [5]. The suppressive effect in the later period is therefore believed to be due to the adaptive immunity, in which immune cells are primed in the manner described above and respond against TAAs.

Overall, the in vivo transfection of ESAT-6 DNA into tumor cells elicits significant inhibition of tumor growth by immediately inducing potent activity of innate immunity mediated by DCs/macrophages in response to the ESAT- 6 signal; this may be followed by adaptive immunity against TAAs. Study is now underway to apply the gene therapy to clinical treatment against cancer in dogs and cats.

ACKNOWLEDGMENTS. Authors acknowledge Prof. Shigeru Kawakami (Nagasaki University), Prof. Yusuke Kono (Ritsumeikan University), and Prof. Mitsuru Hashida (Kyoto University) for their help in measuring the cytokine levels. We also thank Dr. Chieko Yoshihara (Otsuma Women's University) for her help with the experiments. This work was supported by JSPS KAKENHI Grant Numbers 25350555, 16K01394, 26350533, $17 \mathrm{~K} 01390$ and 23380174.

\section{REFERRENCES}

1. Chatterjee, S., Dwivedi, V. P., Singh, Y., Siddiqui, I., Sharma, P., Van Kaer, L., Chattopadhyay, D. and Das, G. 2011. Early secreted antigen ESAT6 of Mycobacterium tuberculosis promotes protective T helper 17 cell responses in a toll-like receptor-2-dependent manner. PLoS Pathog. 7: e1002378. [Medline] [CrossRef]

2. Chen, Z. W., Ahren, B., Ostenson, C. G., Cintra, A., Bergman, T., Möller, C., Fuxe, K., Mutt, V., Jörnvall, H. and Efendic, S. 1997. Identification, isolation, and characterization of daintain (allograft inflammatory factor 1), a macrophage polypeptide with effects on insulin secretion and abundantly present in the pancreas of prediabetic BB rats. Proc. Natl. Acad. Sci. U.S.A. 94: 13879-13884. [Medline] [CrossRef]

3. Cook, E. B., Stahl, J. L., Lowe, L., Chen, R., Morgan, E., Wilson, J., Varro, R., Chan, A., Graziano, F. M. and Barney, N. P. 2001. Simultaneous measurement of six cytokines in a single sample of human tears using microparticle-based flow cytometry: allergics vs. non-allergics. J. Immunol. Methods 254: 109-118. [Medline] [CrossRef]

4. Koyama, Y., Yoshihara, C. and Ito, T. 2015. Novel antitumor strategy utilizing a plasmid expressing a mycobacterium tuberculosis antigen as a "Danger Signal" to block immune escape of tumor cells. Pharmaceutics 7: 165-174. [Medline] [CrossRef]

5. Koyama, Y., Sugiura, K., Yoshihara, C., Inaba, T. and Ito, T. 2015. Highly effective non-viral antitumor gene therapy system comprised of biocompatible small plasmid complex particles consisting of pDNA, anionic polysaccharide, and fully deprotected linear polyethylenimine. Pharmaceutics 7: 152-164. [Medline] [CrossRef]

6. Koyama, Y., Ito, T., Hasegawa, A., Eriguchi, M., Inaba, T., Ushigusa, T. and Sugiura, K. 2016. Exosomes derived from tumor cells genetically modified to express Mycobacterium tuberculosis antigen: a novel vaccine for cancer therapy. Biotechnol. Lett. 38: 1857-1866. [Medline] [CrossRef]

7. Köhler, C. 2007. Allograft inflammatory factor-1/Ionized calcium-binding adapter molecule 1 is specifically expressed by most subpopulations of macrophages and spermatids in testis. Cell Tissue Res. 330: 291-302. [Medline] [CrossRef]

8. Latchumanan, V. K., Singh, B., Sharma, P. and Natarajan, K. 2002. Mycobacterium tuberculosis antigens induce the differentiation of dendritic cells from bone marrow. J. Immunol. 169: 6856-6864. [Medline] [CrossRef]

9. Liu, W., Peng, Y., Yin, Y., Zhou, Z., Zhou, W. and Dai, Y. 2014. The involvement of NADPH oxidase-mediated ROS in cytokine secretion from macrophages induced by Mycobacterium tuberculosis ESAT-6. Inflammation 37: 880-892. [Medline] [CrossRef]

10. Mito, K., Sugiura, K., Ueda, K., Hori, T., Akazawa, T., Yamate, J., Nakagawa, H., Hatoya, S., Inaba, M., Inoue, N., Ikehara, S. and Inaba, T. 2010. IFN $\gamma$ markedly cooperates with intratumoral dendritic cell vaccine in dog tumor models. Cancer Res. 70: 7093-7101. [Medline] [CrossRef]

11. Mustafa, A. S., Shaban, F. A., Al-Attiyah, R., Abal, A. T., El-Shamy, A. M., Andersen, P. and Oftung, F. 2003. Human Th1 cell lines recognize the Mycobacterium tuberculosis ESAT-6 antigen and its peptides in association with frequently expressed HLA class II molecules. Scand. J. Immunol. 57: 125-134. [Medline] [CrossRef]

12. Pathak, S. K., Basu, S., Basu, K. K., Banerjee, A., Pathak, S., Bhattacharyya, A., Kaisho, T., Kundu, M. and Basu, J. 2007. Direct extracellular interaction between the early secreted antigen ESAT- 6 of Mycobacterium tuberculosis and TLR2 inhibits TLR signaling in macrophages. Nat. Immunol. 8: 610-618. [Medline] [CrossRef]

13. Pathan, A. A., Wilkinson, K. A., Wilkinson, R. J., Latif, M., McShane, H., Pasvol, G., Hill, A. V. and Lalvani, A. 2000. High frequencies of circulating IFN-gamma-secreting CD8 cytotoxic T cells specific for a novel MHC class I-restricted Mycobacterium tuberculosis epitope in M. tuberculosis-infected subjects without disease. Eur. J. Immunol. 30: 2713-2721. [Medline] [CrossRef]

14. Schumacher, T. N. and Schreiber, R. D. 2015. Neoantigens in cancer immunotherapy. Science 348: 69-74. [Medline] [CrossRef]

15. Smith, V. L., Cheng, Y., Bryant, B. R. and Schorey, J. S. 2017. Exosomes function in antigen presentation during an in vivo Mycobacterium tuberculosis infection. Sci. Rep. 7: 43578. [Medline] [CrossRef]

16. Trapani, J. A. 2001.Granzymes: a family of lymphocyte granule serine proteases. Genome Biol. 2: Reviews 3014.

17. Travers, P., Walport, M., Shlomchik, M. and Janeway, C. 2001. Basic concepts in immunology. pp. 1-34. In: Immunobiology, 5th ed., Garland Science, New York.

18. Uchida, E., Chambers, J. K., Nakashima, K., Saito, T., Ohno, K., Tsujimoto, H., Nakayama, H. and Uchida, K. 2016. Pathologic features of colorectal inflammatory polyps in Miniature Dachshunds. Vet. Pathol. 53: 833-839. [Medline] [CrossRef]

19. Wang, J., Wang, L., Lin, Z., Tao, L. and Chen, M. 2014. More efficient induction of antitumor T cell immunity by exosomes from CD40L genemodified lung tumor cells. Mol. Med. Rep. 9: 125-131. [Medline] [CrossRef]

20. Wolfers, J., Lozier, A., Raposo, G., Regnault, A., Théry, C., Masurier, C., Flament, C., Pouzieux, S., Faure, F., Tursz, T., Angevin, E., Amigorena, S. and Zitvogel, L. 2001. Tumor-derived exosomes are a source of shared tumor rejection antigens for CTL cross-priming. Nat. Med. 7: $297-303$. [Medline] [CrossRef]

21. Yoshihara, C., Hamada, K., Kuroda, M. and Koyama, Y. 2012. Oncolytic plasmid: A novel strategy for tumor immuno-gene therapy. Oncol. Lett. 3: 387-390. [Medline] 